\title{
BMJ Open Efficacy of pragmatic same-day ring prophylaxis for adult individuals exposed to SARS-CoV-2 in Switzerland (COPEP): protocol of an open-label cluster randomised trial
}

\author{
Mikaela Smit (1) , ${ }^{1,2}$ Annalisa Marinosci, ${ }^{1}$ Giovanni Jacopo Nicoletti, ${ }^{3}$ \\ Thomas Perneger (D) ,2,4 Silvio Ragozzino, ${ }^{5}$ Diego O Andrey, ${ }^{1,2,6}$ Marcel Stoeckle, ${ }^{5}$ \\ Frederique Jacquerioz (1) , ${ }^{7}$ Dan Lebowitz, ${ }^{8,9}$ Thomas Agoritsas, ${ }^{2,10,11}$ \\ Benjamin Meyer, ${ }^{12}$ Herve Spechbach, ${ }^{7}$ Julien Salamun, ${ }^{7}$ Moritz Back, ${ }^{13}$ \\ Carla Schaubhut, ${ }^{13}$ Simon Fuchs, ${ }^{13}$ Laurent Decosterd, ${ }^{14}$ Manuel Battegay, ${ }^{5}$ \\ Idris Guessous, ${ }^{7}$ François Chappuis, ${ }^{7}$ Laurent Kaiser, ${ }^{15,16}$ Niklaus D Labhardt, ${ }^{3,5}$ \\ Alexandra Calmy ${ }^{1,2}$
}

To cite: Smit M, Marinosci A, Nicoletti GJ, et al. Efficacy of pragmatic same-day ring prophylaxis for adult individuals exposed to SARS-CoV-2 in Switzerland (COPEP): protocol of an open-label cluster randomised trial. BMJ Open 2020;10:e040110. doi:10.1136/ bmjopen-2020-040110

- Prepublication history and additional materials for this paper is available online. To view these files, please visit the journal online (http://dx.doi org/10.1136/bmjopen-2020040110).

Received 07 May 2020 Revised 07 0ctober 2020 Accepted 16 0ctober 2020

Check for updates

(c) Author(s) (or their employer(s)) 2020. Re-use permitted under CC BY-NC. No commercial re-use. See rights and permissions. Published by BMJ.

For numbered affiliations see end of article.

Correspondence to

Dr Mikaela Smit;

mikaela.smit@hcuge.ch

\section{ABSTRACT}

Introduction Lopinavir/ritonavir (LPV/r) has been proposed as repurposed drugs for pre-exposure and postexposure prophylaxis as well as therapy of COVID-19. Coronavirus postexposure prophylaxis (COPEP) trial aims at assessing their efficacy as postexposure ring-prophylaxis among adults exposed to SARS-CoV-2.

Methods and analysis COPEP is a two-arm open-label cluster-randomised trial conducted in three cantons of Switzerland. Asymptomatic contacts ( $\geq 16$ years) of individuals diagnosed with COVID-19 will be randomised (2:1) to either LPV/r (400 mg/100 mg two times per day) for 5 days, or a standard of care arm (no treatment). Asymptomatic individuals may be either SARS-CoV-2 positive or negative. Contacts living in the single household will form a cluster and will be randomised into the same arm. All participants will be followed-up for 21 days and undergo daily monitoring for COVID-19 symptoms. The primary endpoint is 21-day incidence of laboratory-confirmed COVID-19 with $\geq 1$ compatible symptom, analysed in an intention-to-treat (ITT) analysis. The secondary endpoints include the 21-day incidence of COVID-19 as well as SARS-CoV-2 infection in a modified ITT analysis, excluding participants who had a positive SARS-CoV-2 RT-PCR from oropharyngeal swab and/or a positive SARS-CoV-2 IgG serology at baseline. Assuming a 21 -day incidence for COVID-19 of 20\% among contacts without postexposure chemoprophylaxis, to detect a relative risk reduction of $60 \%$ (ie, translating in an absolute reduction from $20 \%$ to $8 \%$ ), with a power of $80 \%$, an alpha of $5 \%$. Accounting for design effect of cluster design of circa 1.1, we plan to enrol 200 participants to the LPV/r arm and 100 to the standard of care arm, 300 participants in total.

Ethics and dissemination Ethics approval has been granted by the Commission Cantonale d'Ethique de la Recherche, Ethikkommission Nordwest- und Zentralschweiz and Comitato Etico Cantonale (ref 2020-

\section{Strengths and limitations of this study}

- Among the first clinical trials to study two prophylactic candidates for COVID-19 in the general population as well as healthcare workers

- The trial will test pragmatic ring prophylactic strategies that can be prescribed the same day with minimal clinical and laboratory assessment.

- Recruitment may be slowed down by the evolution of the epidemic.

This is an unblinded trial.

00864) and Swissmedic (2020DR3056). Results from this trial will be disseminated via journal articles and presentations at national and international conferences. Trial registration number Clinicaltrials.gov Registry (NCT04364022); Swiss National Clinical Trial Portal Registry (SNCTP 000003732).

Registered report identifier CCER 2020-0864.

\section{INTRODUCTION}

Since the beginning of the SARS-CoV-2 outbreak in December 2019, the medical and scientific community has scrambled to identify effective pharmacological candidates for its prevention and treatment. COVID-19 (which is the clinical manifestation of SARS-CoV-2 infection) has caused unprecedented pressure on modern health systems across the world. The sudden and uncontrolled influx of severe COVID-19 cases has caused critical shortage of hospital-based resources and forced many countries into lockdown in early 2020. 
Containing the COVID-19 pandemic may necessitate a multipronged strategy, including immunisation, treatment and prophylaxis. The latter would facilitate the containment of future outbreaks of the disease in nonimmunised populations. This current pandemic wave will likely be followed by subsequent outbreaks and authorities will need to consider less strict isolation measures when apprising multiple economic impacts (including on health-related outcome) over a prolonged period of time.

Several drugs have been identified as possible prophylactic candidates. These include the protease inhibitors lopinavir/ritonavir $(\mathrm{LPV} / \mathrm{r})$ used for the treatment of HIV. ${ }^{2}$ Protease inhibitors work by preventing viral replication and LPV/r has been shown to bind to active site of the SARS-CoV protease in vitro, with studies confirming that the spatial structure of the binding site was conserved between SARS-CoV and SARS-CoV-2. ${ }^{3-5} \mathrm{LPV} / \mathrm{r}$ is licensed in most countries, are part of the WHO essential list, and are available globally and at low cost. ${ }^{6}$

However, to date, there are no published randomised studies assessing the efficacy of $\mathrm{LPV} / \mathrm{r}$ as prophylaxis for COVID-19. Coronavirus postexposure prophylaxis (COPEP) is a Swiss two-arm open-label cluster randomised trial that will assess the efficacy, safety and acceptability of same-day LPV/r-based prophylaxis, compared with standard of care alone (no treatment) for asymptomatic individuals exposed to SARS-CoV-2.

\section{METHODS}

COPEP is an open-label two-arm (2:1) cluster randomised superiority trial to assess efficacy, safety and acceptability of same-day LPV/r-prophylaxis vs standard of care (no treatment) for asymptomatic adult individuals exposed to SARS-CoV-2. Asymptomatic individuals may be either SARS-CoV-2 positive or negative. The study will be performed at three sites in Switzerland, Geneva, Basel and Lugano, and seeks to recruit 300 participants over $8-9$ months. The main objective of COPEP is to assess prevention of laboratory-confirmed COVID-19 with $\geq 1$ compatible symptom, with secondary objectives, including preventing of SARS-COV-2 infection and attenuation of COVID-19 severity.

\section{Participants}

This trial enrols individuals ( $\geq 16$ years) who have come into close contact with a confirmed case of SARS-CoV-2 infection. Individuals are eligible to be enrolled if the contact occurred within the last 7 days but no more than 72 hours after the index case was diagnosed. A close contact is defined, as per the Swiss Federal Office of Public Health, as a person who spent $>15 \mathrm{~min}$ in $<2 \mathrm{~m}$ distance or shared closed space with a confirmed case for a prolonged period (eg, more than 2 hours) in the period extending from 48 hours before onset of symptoms (or before the date of testing in the absence of symptoms), or a person who had direct contact with the body fluids or laboratory specimens of a case without recommended personal protective equipment (PPE) or in case of failure of PPE.

Recruitment of participants to this study will be performed through two routes; (1) the Public Health Authorities in Geneva, Basel and Lugano, who, as the standard of care, contact all individuals tested positive for SARS-CoV-2 to provide them with instructions on isolating procedures and self-monitoring and (2) healthcare workers will be recruited via social media platforms and internal hospital-based platforms. All close contacts of confirmed SARS-CoV-2 positive index case will be screened for eligibility (table 1 ).

\section{Intervention}

Participants will be randomised in household clusters, 2:1, to receive either $\mathrm{LPV} / \mathrm{r}, 400 \mathrm{mg} / 100 \mathrm{mg}$ (2× $200 \mathrm{mg} / 50 \mathrm{mg}$ pills) two times per day for 5 days; or standard of care (no treatment). The first dose of $\mathrm{LPV} / \mathrm{r}$ will

Table 1 Inclusion and exclusion criteria

Inclusion criteria Exclusion criteria

1. Documented close contact with a PCRconfirmed SARS-CoV-2 positive individual

2. $\geq 16$ years of age
1. Fever (temperature $>38.0^{\circ} \mathrm{C}$ ) and/or respiratory symptoms (cough, dyspnoea) and/or new anosmia/ageusia

2. Individuals with previous confirmed SARS-CoV-2 infection within the last 6 months

3. Informed consent documented by signature (including parent's or legal guardian's signature if the participant is 16 and 18 years old)

\section{Known impairment of liver function}

4. Known hypersensitivity to any of the study medications

5. Use of any medications that are contraindicated with LPV/r using the website www.hiv-druginteractions.org/checker

6. Individuals on boosted protease inhibitor as part of an antiretroviral therapy

7. Inability to be followed-up for the trial period 
be taken during the baseline visit, as directly observed therapy.

\section{Outcomes \\ Primary endpoint}

The primary endpoint is the 21-day incidence of laboratory-confirmed COVID-19 with $\geq 1$ compatible symptom in individuals exposed to SARS-CoV-2 who are asymptomatic (either SARS-CoV-2 positive or negative) at baseline. The primary analysis includes all individuals enrolled, irrespective of their baseline oropharyngeal swab results or the baseline SARS-CoV-2 serology (intention-to-treat (ITT) analysis).

\section{Secondary endpoints}

- 21-day incidence of COVID-19 in individuals exposed to SARS-CoV-2 who are asymptomatic, and have negative SARS-CoV-2 PCR and serology at baseline (modified ITT).

- 21-day incidence of SARS-CoV-2 infection in individuals exposed to SARS-CoV-2 who are asymptomatic and have negative SARS-CoV-2 PCR and serology at baseline (modified ITT).

- Severity of clinical COVID-19 on a 7-point ordinal scale.

- Incidence of serious adverse events.

\section{Explorative endpoints}

- Acceptability of a prophylaxis for COVID-19.

- Reported adherence to LPV/r for participants on the $\mathrm{LPV} / \mathrm{r}$ arm and HCQ and LPV/r drug levels on day 5 among all individuals.

\section{Measurements}

The following clinical definitions will be used. COVID-19 is defined as $\geq 1$ symptom compatible with COVID-19 (cough, dyspnoea, anosmia, ageusia, elevated temperature $\left.\left(>38.0^{\circ} \mathrm{C}\right)\right)$ and either

1. a positive PCR for SARS-CoV-2 in oropharyngeal swab and/or

2. a seroconversion of IgG only or IgG and IgA for SARS$\mathrm{CoV}-2$ at day 21 in individuals with negative serology at baseline. In case of seroconversion of IgA only, seroconversion of IgG using more sensitive spike-based recombination immunofluorescence assay (S-rIFA) will be assessed.

New SARS-CoV-2 infection is defined as

1. a positive PCR for SARS-CoV-2 (oropharyngeal swab) among those with a negative PCR at baseline and/or

2. a seroconversion of IgG only or IgG and IgA for SARS$\mathrm{CoV}-2$ at day 21 in individuals with negative serology at baseline. In case of seroconversion of IgA only, seroconversion of IgG using more sensitive S-rIFA will be used.

Seroconversion for SARS-CoV-2 is defined as negative results for IgG in ELISA at baseline and either

1. positive result for IgG in ELISA and confirmation by S-rIFA at day 21 or
2. doubtful result for IgG in ELISA at day 21 and confirmation by S-rIFA. In case of negative result for IgG in ELISA at day 21 seroconversion can alternatively be defined as follows: Negative result of IgA in ELISA at baseline and (a) positive or doubtful result for IgA in ELISA at day 21 and (b) positive result for IgG in SrIFA at day 21.

\section{Procedures}

All participants will be followed-up for 21 days (figure 1 and table 2). Participants will undergo an oropharyngeal swab for SARS-CoV-2 PCR and a SARS-CoV-2 serology at baseline and on day 21. In addition, participants will be asked to provide on day 5 , a capillary puncture on dried blood spot (DBS) to assess blood levels of LPV/r. Participants will be given the option of 'self-test', or of a homevisit for this DBS procedure.

During follow-up, individuals will be asked to complete a daily online COVID-19 symptoms questionnaire. The online questionnaire generates alerts when individuals report a symptom associated with COVID-19 and if participants do not complete the questionnaire for 2 consecutive days. The online questionnaire also serves as a reminder for those on the LPV/r arm, to take their daily medication up to day 5 . Paper questionnaires will be made available for those without access to internet. All questionnaires are available in several languages.

The team will contact the participant in case an alert is triggered and do a first clinical assessment by phone. Participants who report COVID-related symptoms (eg, dyspnoea, cough, fever $\left(>38.0^{\circ} \mathrm{C}\right)$, anosmia) will be asked to come onsite for an extra visit and undergo clinical assessment and an oropharyngeal swab to confirm/ exclude SARS-CoV-2 infection. If found positive, participants will be provided with appropriate care, as per local protocol. A follow-up visit 14 days after a positive diagnosis will be conducted, in order to establish the level of clinical manifestation of disease.

On day 21, participants will further complete a questionnaire on adverse events and acceptability of the treatment. Participants on the LPV/r arm are asked to bring the phials which contained the medication to day 21 visit, so that the study team can record the number of returned pills, as per good clinical practice standard.

\section{Sample size}

The sample size for the primary endpoint assumes that without treatment $20 \%$ of close contacts will develop COVID-19, based on the clinical observations made by the team. To detect a relative risk reduction of $60 \%$ (ie, translating in an absolute reduction from $20 \%$ to $8 \%$ ), with a power of $80 \%$, an alpha of $5 \%$ and accounting for design effect of cluster design of circa 1.1 (based on $\mathrm{DE}=(\mathrm{n}-1) \mathrm{r}+1$, where $\mathrm{n}=3$ denotes the average cluster size, and $r=0.05$ is the assumed intraclass correlation coefficient). A sample size of 200 participants on LPV/r and 100 on standard of care arm, or 300 in total will thus be needed. 


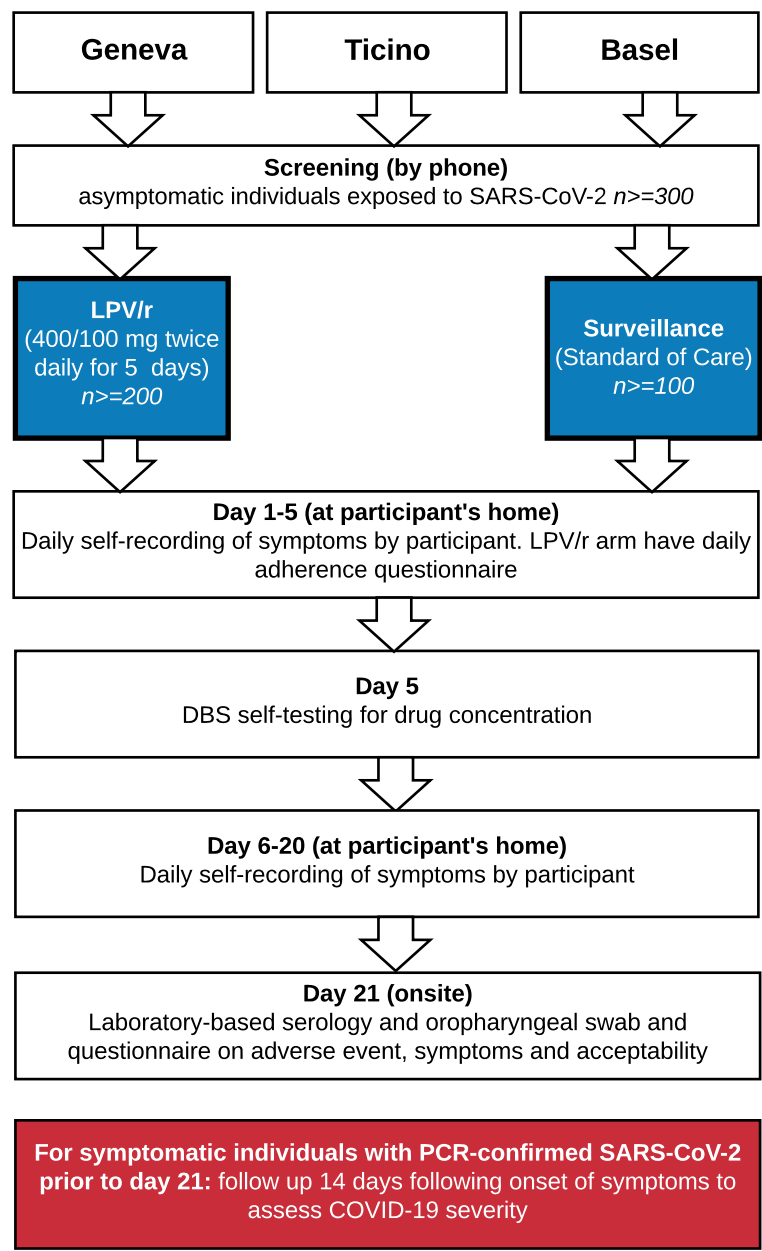

Recruitment: identifying close

contacts of SARS-CoV-2

infected index cases

Screening according to

in/exclusion criteria

Randomisation (day 0 ) onsite:

within 48 hours following the

documented infection of index

case, following informed consent.

Serology and oropharyngeal

swab.

Study period: Day 1 to Day 5

Day 5

Study period: Day 6 to Day 20

Day 21 onsite

Outcome assessment for

infected participants: 14 days

after onset of symptoms

Figure 1 Consolidated Standards of Reporting Trials flow diagram. DBS, dried blood spot; LPV/r, lopinavir/ritonavir.

For the first of the secondary endpoint (occurrence of new SARS-CoV-2 infection) we assume $40 \%$ of close contact without prophylaxis will become infected and $16 \%$ with prophylaxis; but we also expect a baseline prevalence of positive PCR of $30 \%$ (these participants will be excluded from this analysis in a modified ITT). With an effective sample size of 140 individuals on the LPV/r arm $(200 \times 0.7)$ and 70 on the standard of care arm $(100 \times 0.7)$ the power will be $95 \%$ for this endpoint.

\section{Randomisation and blinding}

Randomisation will be done in variable-sized blocks (sizes 6 or 9) in random sequence, stratified by site (Geneva, Basel and Lugano). Randomisation will be done by cluster, that is where close contacts reside in the same household (eg, the family or friends of the index case that live together). The randomisation procedure will consist of sealed envelopes, one for each household cluster, prepared by individuals who are not associated with the recruitment for the trial. Treatment will be identical within households because of the risk of cross-contamination and to simplify treatment administration to individuals in the same household. This is an open-label trial.

\section{Statistical analysis}

For the analysis of the primary endpoint, 21-day incidence of COVID-19, we will perform an ITT, including all individuals who were randomised (including those who will retrospectively be found to be PCR-confirmed SARS-CoV-2 positive at baseline as well as individuals retrospectively found SARS-CoV-2 seropositive by serology).

The intervention arm, LPV/r, will be compared with the surveillance arm, using separate indicator variables for the active treatment arms. Because the hypotheses about treatment efficacy are unrelated and independent, and because we will focus on estimation and CIs rather than on statistical tests, no adjustment will be done for multiplicity. Since individual observations will be clustered within households (randomisation units), themselves nested within index cases, we will use mixed complementary log-log regression models for the main analysis (complementary log-log regression is similar to logistic regression, but yields relative hazards, rather than ORs; relative hazards are more readily interpretable in the context of disease incidence). The outcome variable will be the occurrence of COVID-19 by day 21 . 
Table 2 Standard Protocol Items: Recommendations for Interventional Trials table

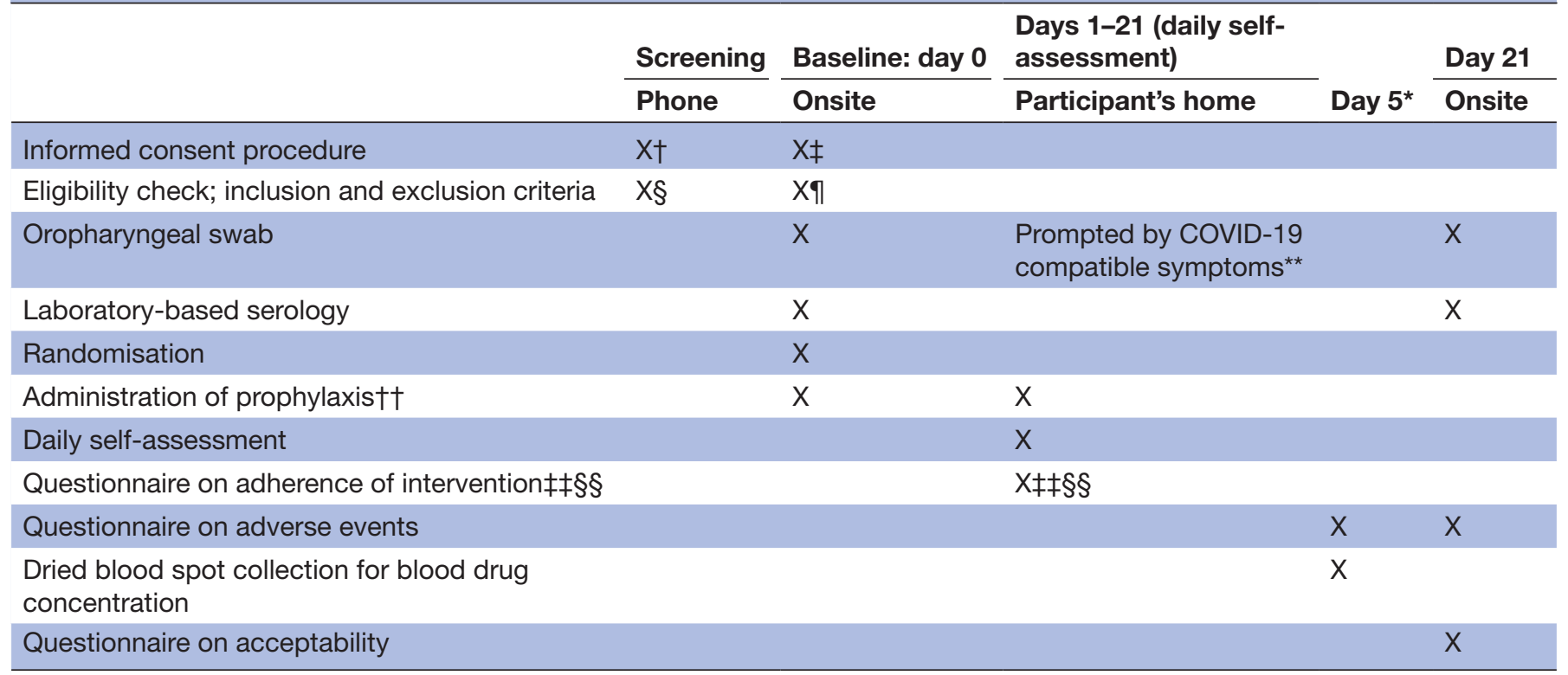

Participants who report COVID-19 symptoms on or prior to day 21; will have onsite visit as soon as possible. An oropharyngeal swab will be performed, and if SARS-CoV-2 is PCR-confirmed, participants will undergo a follow-up visit 14 days after symptoms onset. ${ }^{* *}$

*Participants will be given the option of either a self-test dried blood spot, or home visit by a team member. †Study information will be provided to participants by phone.

łlnformed consent will be signed by participant and medical investigator onsite.

§Eligibility criteria will be checked with participant by phone.

qEligibility criteria will be confirmed.

**Participants with a positive SARS-CoV-2 swab during follow-up will be provided with appropriate care, as per local protocol. ††Only for participants randomised to lopinavir/ritonavir (LPV/r), as directly observed therapy for first dose.

¥¥Only for participants randomised to LPV/r.

$\S \S$ Only between baseline and day 5 .

A random intercept will be defined by each household, nested within the index case. The main fixed effect will be treatment. The main statistical model will be adjusted for potential confounding variables, guided by the most up to date evidence. We foresee that for the incidence of COVID-19, adjustment variables are: age, presence of comorbidities (specifically cardiac, liver or pulmonary disease), treatment of the index case, occupational versus non-occupational exposure and/or positive serology.

In a modified intent to treat analysis, we will evaluate the secondary endpoints; (1) 21-day incidence of COVID-19 in individuals exposed to SARS-CoV-2 who are asymptomatic, PCR-confirmed SARS-CoV-2 negative and have negative SARS-CoV-2 serology at baseline (modified ITT), (2) 21-day incidence of SARS-CoV-2 infection in individuals exposed to SARS-CoV-2 who are asymptomatic, PCR-confirmed SARS-CoV-2 negative and have negative SARS-CoV-2 serology at baseline (modified ITT). The output will be an adjusted relative hazard of the treatment effect versus surveillance, with a $95 \%$ CI.

For the secondary outcome 'severity of disease', rated on a 7-point scale, we will use a Mann-Whitney test to compare this ordinal outcome variable between intervention arm and surveillance. For the analysis of individualreported adverse events, the population will include all randomised participants, and the comparisons will be by cross-tabulations and $\chi^{2}$ tests, since the probability of experiencing adverse events will likely not be affected by clustering. For the analysis of acceptability/compliance, only the intervention arm will be evaluated. We will use R software V.1.2.5019 and Stata software V.16 for the analysis.

\section{Data collection and management}

Any data collected during the course of this study will be protected under secure password, or kept under lock and key in the research office. No records bearing patient identification will be provided to anyone outside of the institution except regulatory agencies. Patients will not be identifiable as individuals in any publication or presentation of this study. Data will be recorded in RedCap and analysed as described above. The trial will follow all standard procedures, including reporting of adverse events. The protocol was written in accordance with Standard Protocol Items: Recommendations for Interventional Trials checklist (see online supplemental appendix 1)

\section{Patient and public involvement}

No patient or patient advisor was involved with study design, recruitment or conduct. 


\section{ETHICS AND DISSEMINATION}

The study (protocol v3 09.06.2020) has been approved by the following boards: Commission Cantonale d'éthique de la Recherche, Geneva, Switzerland (2020-00864), Ethikkommission Nordwest- und Zentralschweiz, Comitato Etico Cantonale and Swissmedic (Swiss Agency for Therapeutic Products).

All participants will be asked for written informed consent with a dedicated member of the research team. This trial will be conducted in accordance with good clinical practice and the Helsinki Declaration. The WHO Trial Registration Data Set is in online supplemental appendix 2.

The COPEP-trial will establish whether $\mathrm{LPV} / \mathrm{r}$ is effective as postexposure chemoprophylaxis against clinical COVID-19 and/or SARS-CoV-2 infection compared with surveillance among asymptomatic individuals with recent contact to a person infected with SARS-CoV-2. Furthermore, this trial will aim to investigate whether LPV/r reduces the severity of clinical COVID-19 over surveillance alone, and whether these prophylaxes are safe and acceptable for postexposure prophylaxis of SARS-CoV-2 and COVID-19.

If effective, such a pragmatic approach with prescription the same day and without waiting for laboratory results, will provide a feasible and relatively low-cost strategy to contain local COVID-19 outbreaks in future. All results will be disseminated in peer-reviewed journals and national and international conferences.

\section{Author affiliations}

${ }^{1}$ HIV Unit, Geneva University Hospitals, Geneva, Switzerland

${ }^{2}$ Faculty of Medicine, University of Geneva, Geneva, Switzerland

${ }^{3}$ Department of Medicine, Swiss Tropical and Public Health Institute, Basel,

Switzerland

${ }^{4}$ Division of Clinical Epidemiology, Geneva University Hospitals, Geneva, Switzerland

${ }^{5}$ Department of Infectious Diseases and Hospital Epidemiology, University of Basel,

Basel, Switzerland

${ }^{6}$ Division of Laboratory Medicine, Diagnostic Department, Geneva University Hospitals, Geneva, Switzerland

${ }^{7}$ Department of Primary Care, Geneva University Hospitals, Geneva, Switzerland

${ }^{8}$ Infection Control Programme, Geneva University Hospitals, Geneva, Switzerland

${ }^{9}$ Direction Generale de la Sante, Republique et Canton de Geneve, Geneva,

Switzerland

${ }^{10}$ Department of Medicine, Geneva University Hospitals, Geneva, Switzerland

${ }^{11}$ Department of Health Research Methods, Evidence, and Impact, McMaster

University, Hamilton, Ontario, Canada

${ }^{12}$ Centre for Vaccinology, Department of Pathology and Immunology, University of Geneva, Geneva, Switzerland

${ }^{13}$ Gesundheitsdepartement, Canton of Basel City, Basel, Switzerland

${ }^{14}$ Laboratory of Clinical Pharmacology, University of Lausanne, Lausanne,

Switzerland

${ }^{15}$ Division of Infectious Diseases, Geneva University Hospitals, Geneva, Switzerland

${ }^{16}$ Geneva Centre for Emerging Viral Diseases, Geneva University Hospitals, Geneva, Switzerland
Twitter Frederique Jacquerioz @FrederiqueJ

Contributors $\mathrm{AC}$ and NDL conceived the overall trial, including the overall design and therapeutic candidates and are the PI and Co-PI. AC, NDL, MSm, TP, MSt and AM designed the trial, including decision on intervention dosage and administration, study endpoints and procedures. MSm and TP developed all statistical aspects of the trial design. MSm will perform the trial data analysis, with the support of TP. DL and SF contributed to the formulation of recruitment via the cantonal health authorities, together with MBack and CS. AM and DOA developed the patient and laboratory pathways. BM, LK and LD helped with all laboratory aspects of the trial design. MSm wrote the first draft of this manuscript. GJN, SR, AM, MSt, NDL and AC are the study physicians and together with FJ, TA, HS, JS, MBattegay, IG and FC who manage the respective patients with COVID-19 or infectious diseases unit contributed to the patient pathway. All authors contributed to the redrafting of the manuscript and in the process of approving the final draft.

Funding Fondation privée des HUG (no grant number), Swiss National Fund (project number: 33IC30_166819).

Competing interests None declared.

Patient consent for publication Not required.

Provenance and peer review Not commissioned; externally peer reviewed.

Supplemental material This content has been supplied by the author(s). It has not been vetted by BMJ Publishing Group Limited (BMJ) and may not have been peer-reviewed. Any opinions or recommendations discussed are solely those of the author(s) and are not endorsed by BMJ. BMJ disclaims all liability and responsibility arising from any reliance placed on the content. Where the content includes any translated material, BMJ does not warrant the accuracy and reliability of the translations (including but not limited to local regulations, clinical guidelines, terminology, drug names and drug dosages), and is not responsible for any error and/or omissions arising from translation and adaptation or otherwise.

Open access This is an open access article distributed in accordance with the Creative Commons Attribution Non Commercial (CC BY-NC 4.0) license, which permits others to distribute, remix, adapt, build upon this work non-commercially, and license their derivative works on different terms, provided the original work is properly cited, appropriate credit is given, any changes made indicated, and the use is non-commercial. See: http://creativecommons.org/licenses/by-nc/4.0/.

\section{ORCID iDs}

Mikaela Smit http://orcid.org/0000-0001-8530-748X

Thomas Perneger http://orcid.org/0000-0001-5667-0968

Frederique Jacquerioz http://orcid.org/0000-0002-6957-0920

\section{REFERENCES}

1 European AIDS Clinical Society. Guidelines, 2019. Available: http:// www.europeanaidsclinicalsociety.org/index.php?option=com content\&view $=$ article\&id $=59 \&$ Itemid $=41$

2 World Health Organization. Antiretroviral therapy for HIV infection in adults and adolescents. Available: http://www.who.int/hiv/pub/arv/ adult/en/index.html

3 Anand K, Palm GJ, Mesters JR, et al. Structure of coronavirus main proteinase reveals combination of a chymotrypsin fold with an extra alpha-helical domain. Embo J 2002;21:3213-24.

4 Lin C-W, Tsai C-H, Tsai F-J, et al. Characterization of trans- and ciscleavage activity of the SARS coronavirus 3CLpro protease: basis for the in vitro screening of anti-SARS drugs. FEBS Lett 2004;574:131-7.

5 Harrison C. Coronavirus puts drug repurposing on the fast track. Nat Biotechnol 2020;38:379-81.

6 World Health Organization. WHO model lists of essential medicines. Available: http://www.who.int/medicines/publications/ essentialmedicines/en/ 